\title{
Liquids on Topologically Nanopatterned Surfaces
}

\section{Citation}

Gang, Oleg, Kyle J. Alvine, Masafumi Fukuto, Peter S. Pershan, Charles T. Black, and Benjamin M. Ocko. 2005. Liquids on topologically nanopatterned surfaces. Physical Review Letters 95(21): 217801.

\section{Published Version}

doi:10.1103/PhysRevLett.95.217801

\section{Permanent link}

http://nrs.harvard.edu/urn-3:HUL.InstRepos:4239019

\section{Terms of Use}

This article was downloaded from Harvard University's DASH repository, and is made available under the terms and conditions applicable to Other Posted Material, as set forth at http:// nrs.harvard.edu/urn-3:HUL.InstRepos:dash.current.terms-of-use\#LAA

\section{Share Your Story}

The Harvard community has made this article openly available.

Please share how this access benefits you. Submit a story.

Accessibility 


\title{
Liquids on Topologically Nanopatterned Surfaces
}

\author{
Oleg Gang, ${ }^{1,2, *, \dagger}$ Kyle J. Alvine, ${ }^{3}$ Masafumi Fukuto, ${ }^{3}$ Peter S. Pershan, ${ }^{3, *, *}$ Charles T. Black, ${ }^{4}$ and Benjamin M. Ocko ${ }^{1, *, \S}$ \\ ${ }^{1}$ Department of Physics, Brookhaven National Laboratory, Upton, New York 11973, USA \\ ${ }^{2}$ Center for Functional Nanomaterials, Brookhaven National Laboratory, Upton, New York 11973, USA \\ ${ }^{3}$ DEAS, Harvard University, Cambridge, Massachusetts 02138, USA \\ ${ }^{4}$ IBM T. J. Watson Research Center, Yorktown Heights, New York 10598, USA
}

(Received 4 February 2005; published 16 November 2005)

\begin{abstract}
We report here surface $\mathrm{x}$-ray scattering studies of the adsorption of simple hydrocarbon liquid films on nanostructured surfaces - silicon patterned by an array of nanocavities. Two different regimes, filling and growing, are observed for the wetting film evolution as a function of the chemical potential offset $\Delta \mu$ from the bulk liquid-vapor coexistence. The strong influence of geometrical effects is manifested by a $\Delta \mu$ dependence of liquid adsorption $\Gamma$ in the nanocavities that is stronger than the van der Waals behavior $\Gamma \sim \Delta \mu^{-1 / 3}$ for flat surfaces. The observed $\Delta \mu$ dependence is, however, much weaker than predicted for the infinitely deep parabolic cavities, suggesting that the finite-size effects contribute significantly to the observed adsorption behavior.
\end{abstract}

DOI: 10.1103/PhysRevLett.95.217801

The behavior of liquids on laterally heterogeneous surfaces has recently attracted much theoretical attention [14] due to its relevance to the basic properties of liquids that are confined to nanoscale structures $[5,6]$. Related phenomena such as the effect of nanoroughness on wetting [7] and the crossover to capillary filling [8] have impact on emerging applications, including nanofluidic devices [9], nanotemplating [10], and surface rheology [11]. For homogeneous flat surfaces, the microscopic development of liquid wetting films is dictated by the details of the intermolecular interactions $[12,13]$. The power-law form $d \propto$ $\Delta \mu^{-\beta}$ often successfully describes the dependence of film thickness $d$ on the chemical potential offset $\Delta \mu$ from the bulk liquid-vapor equilibrium. For flat surfaces and van der Waals (vdW) forces the exponent $\beta=1 / 3$ [14]. Much more complex behavior is found for random rough or self-affine surfaces $[15,16]$, due to the contribution of the microscopic local curvature [17]. However, for welldefined surface topologies, recent theoretical studies predict a power-law form dependence for liquid adsorption with $\beta \neq 1 / 3$. The direct correspondence between the wetting exponent $\beta$ and the shape of surface structures can be established theoretically for a variety of geometries [18-21]. On the other hand, experimental constraints have limited the verification of these predictions to isolated submicron drops [22] and micron-sized surface topographical features $[23,24]$, and the majority of the predicted phenomena on the nanoscale has remained untested.

In this Letter we report the microscopic evolution of the wetting film of methyl-cyclohexane (MCH) on silicon surfaces patterned with nanoscale arrays of parabolically shaped cavities. We demonstrate that the nanoscale wetting behavior of thin liquid films, $1-20 \mathrm{~nm}$ thick, can be studied by probing nanopatterned surfaces using x-ray reflectivity (XR) and grazing incidence diffraction (GID), both well developed quantitative techniques [25]. At small $\Delta \mu$, where a liquid film completely covers the surface patterns,
PACS numbers: 68.15.+e, 61.30.Hn, 68.03.Fg, 68.08.Bc

we observed the $\Delta \mu^{-1 / 3}$ dependence for the adsorption, similar to the vdW interacting film on flat surface. At larger $\Delta \mu$, the adsorption behavior on patterned surface crosses over to a geometry dominated dependence with steeper
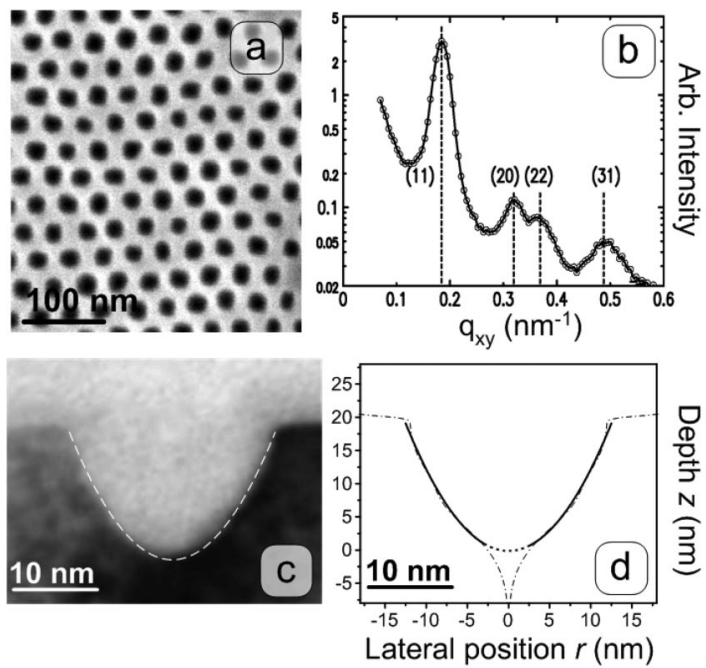

FIG. 1. (a) Scanning electron microscopy (top view) of the hexagonally packed array of nanocavities. (b) GID pattern of the hexagonal surface array. (c) TEM cross section of the individual cavity. The cavity depth is $17.2 \pm 0.8 \mathrm{~nm}$ and the diameter at opening is $24.6 \pm 2 \mathrm{~nm}$ (averaged over 5 pits). (d) The cavity shape (solid line) reconstructed from the electron-density profile $\rho_{\text {dry }}(z)$ as $r(z)=\sqrt{\left(1-\left\langle\rho_{\text {dry }}(z)\right\rangle / \rho_{\text {Silicon }}\right) A / \pi}$ (dot-dashed line), where $A=(\sqrt{3} / 2) c^{2}$ is the hexagonal unit cell area and $c=$ $39.4 \mathrm{~nm}$ is the nearest neighbor distance. This curve is also shown in (c) as the dashed line. Since XR is not sensitive to the structures near the bottom of the cavity, the power-law shape discussed in the text was only fitted to $r(z)$ in the range $2.3 \mathrm{~nm}<$ $r<12 \mathrm{~nm}$. The region for $r<2.3 \mathrm{~nm}$ (dotted line) was interpolated. 
than the $1 / 3$ power law $\left(\sim \Delta \mu^{-0.76}\right.$ ), where the filling of surface cavities occurs.

A nanopatterned substrate was prepared by reactive ion etching of an underlying silicon substrate coated with a self-assembled block-copolymer mask [26]. The resulting hexagonal array, shown in Fig. 1(a), consists of $\sim 20 \mathrm{~nm}$ diameter cavities, with nearest neighbor distances of about $40 \mathrm{~nm}$. The peaks observed in the GID intensity [Fig. 1(b)] as a function of wave vector transfer in the surface plane, $q_{x y}$, are consistent with hexagonal packing of the cavities. From transmission electron microscopy (TEM) studies it is apparent that the cross section of the dry cavities is approximately parabolic [Fig. 1(c)]. Since TEM is not a practical method for volatile liquids, the wet cavities are probed by x-ray methods. One measure of the reliability of the x-ray approach can be obtained by a comparison between the TEM result and the pit shape [Fig. 1(d)] extracted from the XR described below [Fig. 3(a)]. In the region from the cavity bottom $(r=z=0)$ to the flat substrate $(z \sim 20 \mathrm{~nm})$ the shape of the surface defining the cavity can be conveniently parameterized by $z \approx$ $r^{\gamma} / L^{\gamma-1}$ [1], where the best fit to the XR yields the characteristic length, $L=8 \pm 1.2 \mathrm{~nm}$, and $\gamma=$ $2.0 \pm 0.4$. The TEM and XR derived shapes are in agreement at $r>2.3 \mathrm{~nm}$. The cusplike difference at $r<2.3 \mathrm{~nm}$ may originate from a distribution of cavity depths where the corresponding volume difference is small [Fig. 1(d)].

To achieve liquid films with a nanometer scale thickness, the liquid adsorption was varied by controlling the chemical potential difference between the adsorbed film and a reservoir of bulk liquid, $\Delta \mu=\partial \mu_{0} /\left.\partial T\right|_{p} \Delta T \approx$ $-H \Delta T / T$, where $\mu_{0}(P, T)$ is the chemical potential of the bulk liquid, $T=305 \mathrm{~K}$ and $H=5.8 \times 10^{-20} \mathrm{~J}$ is the latent heat of vaporization per $\mathrm{MCH}$ molecule [14]. Temperature differences $\Delta T$ of $1 \mathrm{mK}$ to $10 \mathrm{~K}$ between the substrate and the reservoir provide thickness control from $20 \mathrm{~nm}$ to sub-nm over the macroscopic area [27].

In addition to confirming the hexagonal packing of the pattern, GID probes the average form factor of the cavities, and is therefore sensitive to decreases in electron-density contrast between the cavity and the silicon as the cavity

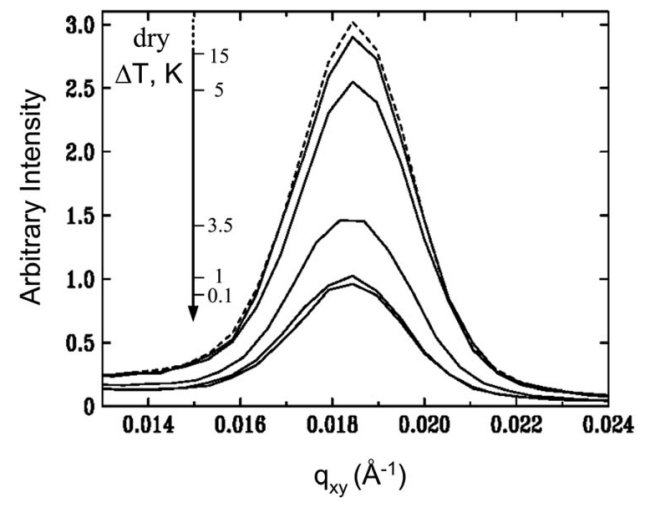

FIG. 2. The (11) GID scattering profiles vs $q_{x y}$ measured at the values of $\Delta T$ shown in the scale. fills with liquid. As shown in Fig. 2, for $15 \mathrm{~K}>\Delta T>$ $0.5 \mathrm{~K}$, there is a systematic reduction in the intensity of (11) hexagonal diffraction peak as the liquid adsorption $\Gamma_{c}$ increases. For $\Delta T<0.5 \mathrm{~K}$, the cavities are completely filled and no further intensity changes have been observed. A reasonable measure of the liquid filling can be obtained from $\Gamma_{c}(\Delta T) \propto \sqrt{I_{\text {dry }}}-\sqrt{I(\Delta T)}$, where $I_{\text {dry }}$ and $I(\Delta T)$ are the integrated intensities of the (11) peak for the dry and wet surfaces.

Details of the surface normal structure of the wetting film have been probed by XR, in which the specular intensity is measured as function of the surface normal wave vector transfer, $q_{z}$ [25]. Figure 3(a) shows representative XR data from the patterned substrate for the dry (black) and wet surfaces (red and blue) at $\Delta T$ between 0.001 and $15 \mathrm{~K}$, along with calculated model fits. The weak modulations at low $q_{z}<0.1 \AA^{-1}$ are due to interference between scattering from the flat top surface and the cavity bottom. The depth of the cavity corresponds to approximately $D \approx 2 \pi / \Delta q_{z} \approx 20 \mathrm{~nm}$, where $\Delta q_{z} \approx 0.03 \AA^{-1}$ is the period of the XR modulation. The changes at $q_{z}<$ $0.1 \AA^{-1}$ in the presence of vapor are related to liquid adsorption in the cavity, whereas the modulations in the
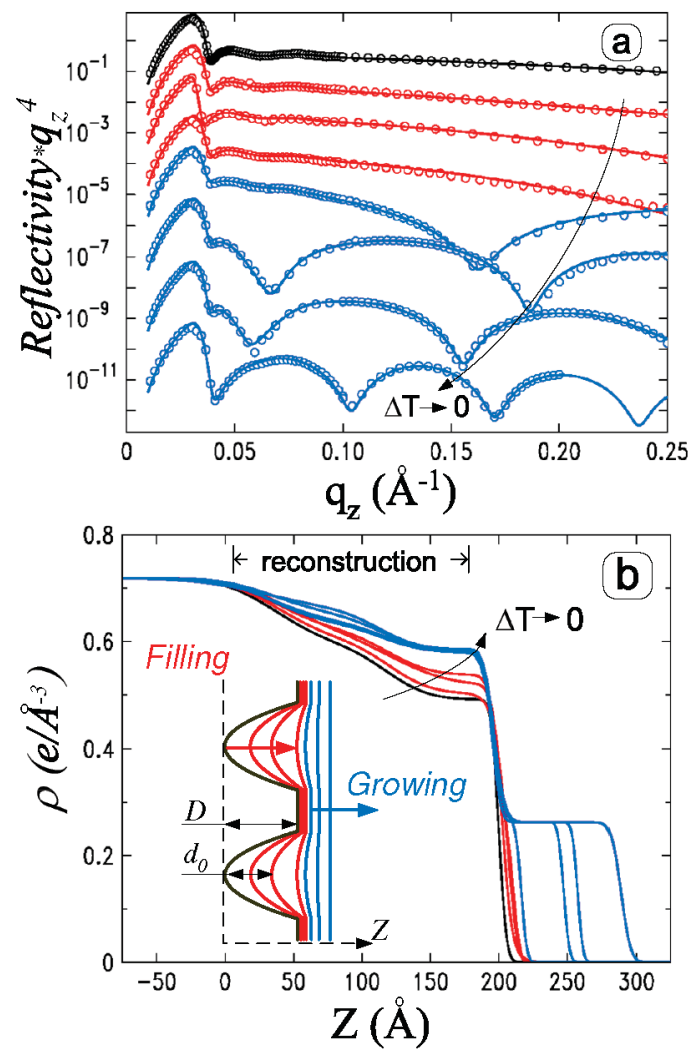

FIG. 3 (color). (a) XR data for the dry (black circles) and wet (red and blue circles) obtained on the nanopatterned surface and the corresponding fits (lines) as described in the text. (b) Corresponding electron-density profiles for the dry (black) and wet surfaces, in the filling (red) and growing (blue) regimes. Inset: Schematic representation of the regimes. 
$\mathrm{XR}$ at larger $q_{z}$ are inversely proportional to the thickness of film on the flat regions of the substrate. The best representations of the average electron-density profiles over the hexagonal unit cell, $\langle\rho(z)\rangle$, are obtained by least-squares fitting of the $\langle\rho(z)\rangle$ dependent XR to the measured XR in the dynamic limit [28].

The simplest model of the dry cavity is the abovementioned quadratic parameterization $(\gamma=2)$. In the range $0<z \leq D$, the cavity's parabolic shape yields an average electron density $\left\langle\rho_{\text {dry }}(z)\right\rangle \propto z$. Further analysis was done with a more flexible $\langle\rho(z)\rangle$ model that was composed of 2 slabs of variable density which are smeared by roughness factors. The cavity part $(0<z<D)$ of the black curve in Fig. 3(b) that represents the best fit to the dry surface is reasonably close to the simple linear density profile expected from the parabolic shape. For the wet surface the thicknesses of the 2 slabs were fixed at the values determined for the dry cavity and the liquid adsorption in the cavity was modeled by their densities. A third slab was added to describe the adsorption above the cavity opening.

The best-fit electron-density models are shown in Fig. 3(b) for decreasing $\Delta T$ from 10 to $0.001 \mathrm{~K}$. As $\Delta T$ is reduced from 10 to $0.5 \mathrm{~K}$ there is a continuous increase in $\langle\rho(z)\rangle$ within the cavity region $[0<z<D$ in Fig. 3(b), red], with little corresponding change in the $\langle\rho(z)\rangle$ above the surface. In contrast, when $\Delta T$ is decreased further, there is a monotonic growth of the liquid film on the silicon surface $[z>D$ in Fig. 3(b), blue] from $1.4 \mathrm{~nm}$ at $0.5 \mathrm{~K}$ to $7.5 \mathrm{~nm}$ at $1 \mathrm{mK}$ [see Fig. 4(b)] with small changes in $\langle\rho(z)\rangle$ in the cavity region. Thus, two distinct regimes of the liquid film development are apparent from the profiles, i.e., filling and growing. When the cavities are completely filled, the electron density has increased by $0.09 e / \AA^{3}$ at $z=D$. Since the opening of cavities covers $34 \%$ of the surface area, this corresponds to an effective fluid electron density of $0.26 \pm 0.01 e / \AA^{3}$, close to $\rho=0.26 e / \AA^{3}$ of bulk MCH.

The dependence of the wetting films on $\Delta \mu$ has been predicted to be remarkably different for the infinitely deep wedge as compared to a flat surface [1,2]. In order to compare different behaviors it is convenient to introduce the areal adsorption, $\Gamma=\left\langle\int_{0}^{\infty}\left(\rho(z)-\rho_{\text {dry }}(z)\right) d z\right\rangle$. vdW adsorption on flat surfaces yields a uniform film of thickness $d=\Gamma / \rho$, and $\Gamma(\Delta T) \propto d \propto \Delta T^{-1 / 3}[29,30]$. Indeed, the thickness (from 0.8 to $12 \mathrm{~nm}$ ) on the flat surface follows the $1 / 3$ power law, as shown in Fig. 4(b), right scale. For patterned surfaces, the amount of liquid per unit cell can be expressed as the sum $\Gamma=\Gamma_{c}+\Gamma_{t}$ of the amount adsorbed in the cavity, $\Gamma_{c}=\left\langle\int_{0}^{D}\left(\rho(z)-\rho_{\mathrm{dry}}(z)\right) d z\right\rangle$, and the amount adsorbed above the cavity opening, $\Gamma_{t}=$ $\left\langle\int_{D}^{\infty} \rho(z) d z\right\rangle$ [Fig. 3(b), inset]. For very deep isolated cavities, the theoretical prediction for the adsorption of liquid is that it should follow the functional form [18], $\Gamma_{c} \propto$ $\Delta T^{-\beta_{c}}$, with a geometry dependent exponent $\beta_{c}$. For a concave wedge, the thickness of liquid in the center $d_{0}$ is predicted to follow $d_{0} \propto \Delta T^{-\gamma}[1,18]$, where $\gamma=2$ in our
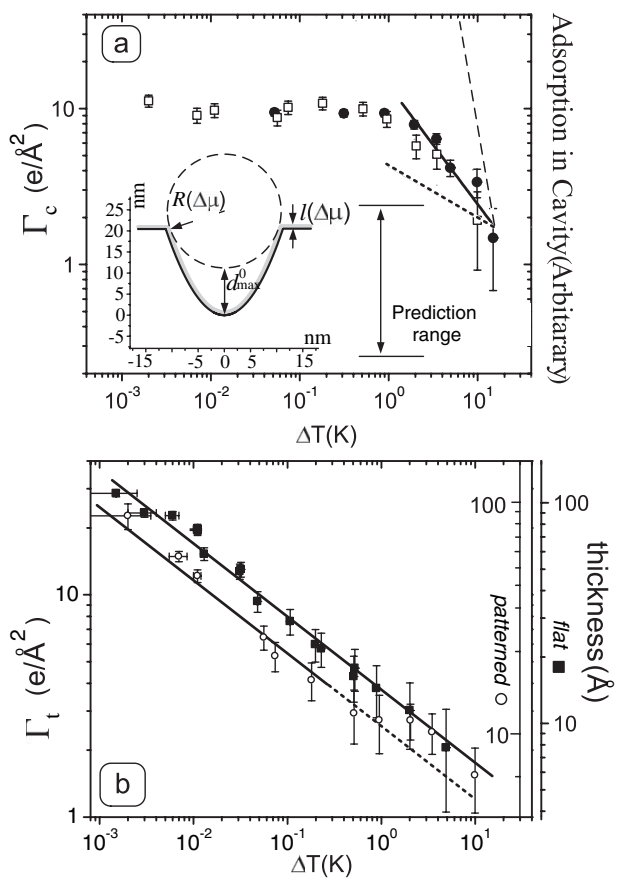

FIG. 4. (a) Liquid adsorption within the cavity obtained from XR $(\square)$ and GID ( $\left(-\right.$ ). Black line represents $\beta_{c}=0.76$, dotted and dashed lines correspondingly represent $\beta=1 / 3$ and $\beta=$ 3.4. The $\Gamma$ range for which the theory for infinitely deep cavities applies [1] is estimated from the geometrical construction (inset). Surface is covered with a wetting layer (gray) of thickness $l(\Delta \mu)$, drawn to scale. (b) Liquid adsorption $\Gamma_{t}$ on the patterned substrate above the flat part $(\bigcirc)$ and on a planar substrate $(\mathbf{\square})$. Fit (solid lines) yields $\beta=1 / 3$ for $\Delta T<0.5 \mathrm{~K}$; for $\Delta T>0.5 \mathrm{~K}$ the deviation from the extrapolation of the $\beta=1 / 3$ law (dashed line) is observed for the patterned substrate.

case. The $\Delta T$ dependence of liquid adsorption can be roughly estimated by using this relation for $d_{0}$ and accounting for the volume $V\left(d_{0}\right)=(\pi / 2) L d_{0}^{2}+(\pi / 3)\left(L d_{0}\right)^{3 / 2}$ between the parabolic solid cavity and a spherical liquid surface [1,18], where for the liquid with the number density $\rho_{n}$ and surface tension $\sigma$ the radius, $R=2 \sigma / \Delta \mu \rho_{n}$ [inset to Fig. 4(a)] is given by the Laplace equation. For the experimentally relevant cavity size this can be well approximated as $\Gamma_{c} \propto V \sim \Delta T^{-3.4}$.

An experimental measure of $\beta_{c}$ can be obtained from GID analysis of $\Gamma_{c}(\Delta T)$ [Figs. 2 and 4(a), 0 ] in the regime when the cavities are filling [Fig. 4(a), $\square$ ]. $\Gamma_{c}(\Delta T)$ estimated from the XR [Fig. 4(a), $\square$ ] demonstrates similar behavior; however, due to the large error bar $(\sim 20 \%)$ in the XR measure of $\Gamma_{c}(\Delta T)$, it is not possible to obtain the detailed temperature dependence from the XR alone. The measured values for $\Gamma_{c}$ can most easily be compared with the predicted behaviors for the two limiting cases, i.e., flat surfaces with vdW interaction $\left(\beta_{c}=1 / 3\right)$ and isolated, infinitely deep parabolic cavities $\left(\beta_{c} \sim 3.4\right)$, by recognizing that the observed $\Gamma_{c}$ from the GID in the filling regime $(1 \mathrm{~K}<\Delta T<10 \mathrm{~K})$ is reasonably well represented by the power-law form $\Gamma_{c} \propto \Delta T^{-\beta_{c}}$ with 
$\beta_{c}=0.76-0.2 /+0.4$ [Fig. 4(a), line]. This clearly demonstrates that the wetting of the cavities varies more rapidly than the $\beta_{c}=1 / 3$ of the flat surface. On the other hand, the observed dependence is weaker than the predicted $\beta_{c} \sim 3.4$ behavior. The theoretical prediction for the deep cavities [1] should apply for $d_{0}<d_{0}^{\max }$ where a sphere of radius $R$ (described above) can be inscribed into the cavity. From a simple geometrical construction [Fig. 4(a) inset] we estimate $d_{0}^{\max }$ is $10 \mathrm{~nm}$, corresponding to $\Gamma_{c}<2.7 e / \AA^{2}$. This indicates that for most of the filling regime the finite size and truncation of the cavities should not be neglected, and hence it is not surprising that the rate of filling is significantly different than expected for deep isolated cavities. At present there is no quantitative theory that fully accounts for the finite-size effects and nonlocal liquid-surface interactions [31].

In Fig. 4(b) we compare the behavior of liquid adsorption $\Gamma(\Delta T)$ on a flat substrate $(\boldsymbol{\square})$ and on the patterned surface $(\bigcirc)$ above the cavity opening $(z>20 \mathrm{~nm})$ over the full measured region $10 \mathrm{~K} \geq \Delta T>0.001 \mathrm{~K}$. Adsorption on the flat surface is in excellent agreement with the $1 / 3$ power-law dependence (solid line) over the entire $\Delta T$ range. For the patterned surface the dependence cannot be described by a simple power law over the full range. For $\Delta T<0.5 \mathrm{~K}$, the wetting behavior is similar to that of the flat surface, albeit with a smaller thickness (solid line). For $\Delta T>0.5 \mathrm{~K}$, where the cavity filling occurs (dashed line), the thickness of the liquid layer on the top surface appears to be close to the values observed for the flat substrate. The observed trend is similar to the theoretical results of Robbins et al. [17] for 2D rectangular geometries.

In summary, we have demonstrated that an array of nanocavities dramatically modifies the wetting behavior in the regime where the cavities are filled as compared to the $1 / 3$ power-law dependence observed on flat surfaces. In this "filling regime" the observed $\Delta \mu$ dependence of liquid adsorption is considerably weaker than that predicted for deep parabolic cavities. The results suggest that finite-size effects considerably influence the wetting behavior of nanoscale cavities, which, for deep cavities, was predicted to be uniquely dependent on the cavity shape only $[1,18]$. Studies of other surface geometries as well as refined theoretical descriptions and simulations that account for the actual cavity size should provide further insights into wetting phenomena on nanostructured surfaces.

Support by U.S. DOE Contract No. DE-AC-0298CH10886, and NSF Grants No. DMR-0124936 and No. 0303916 is gratefully acknowledged. We thank A. Checco and A. Gibaud for helpful discussion and W. Moberly Chan for the TEM help.

\footnotetext{
*Authors to whom correspondence should be addressed.

†Email address: ogang@bnl.gov

‡Email address: pershan@deas.harvard.edu

${ }^{\S}$ Email address: ocko@bnl.gov
}

[1] C. Rascon and A. O. Parry, Nature (London) 407, 986 (2000).

[2] K. Rejmer, S. Dietrich, and M. Napiorkowski, Phys. Rev. E 60, 4027 (1999).

[3] A. Milchev, M. Muller, K. Binder, and D. P. Landau, Phys. Rev. E 68, 031601 (2003).

[4] P. Lenz and R. Lipowsky, Phys. Rev. Lett. 80, 1920 (1998).

[5] S. Dietrich, J. Phys. Condens. Matter 10, 11469 (1998).

[6] T. Becker and F. Mugele, Phys. Rev. Lett. 91, 166104 (2003).

[7] D. Quere, Physica (Amsterdam) 313A, 32 (2002).

[8] L. D. Gelb, Mol. Phys. 100, 2049 (2002).

[9] H. Cao, Z. N. Yu, J. Wang, J. O. Tegenfeldt, R. H. Austin, E. Chen, W. Wu, and S. Y. Chou, Appl. Phys. Lett. 81, 174 (2002).

[10] L. J. Guo, J. Phys. D 37, R123 (2004).

[11] G. McHale, N. J. Shirtcliffe, S. Aqil, C. C. Perry, and M. I. Newton, Phys. Rev. Lett. 93, 036102 (2004).

[12] S. Dietrich, in Phase Transitions and Critical Phenomena, edited by C. Domb and J. Lebowitz (Academic, New York, 1988), Vol. 12.

[13] M. Schick, in Proceedings of the Les Houches Summer School of Theoretical Physics Session XLVIII, edited by J. Charvolin, J.-F. Joanny, and J. Zinn-Justin (Elsevier, Amsterdam, 1990).

[14] I. M. Tidswell, T. A. Rabedeau, P.S. Pershan, and S. D. Kosowsky, Phys. Rev. Lett. 66, 2108 (1991).

[15] P. Pfeifer, Y. J. Wu, M. W. Cole, and J. Krim, Phys. Rev. Lett. 62, 1997 (1989).

[16] M. Kardar and J. O. Indekeu, Europhys. Lett. 12, 161 (1990).

[17] M. O. Robbins, D. Andelman, and J.-F. Joanny, Phys. Rev. A 43, 4344 (1991).

[18] C. Rascon and A. O. Parry, J. Chem. Phys. 112, 5175 (2000).

[19] C. Rascon and A. O. Parry, Phys. Rev. Lett. 94, 096103 (2005).

[20] E. Cheng and M. W. Cole, Phys. Rev. B 41, 9650 (1990).

[21] P. J. Upton, J. O. Indekeu, and J.M. Yeomans, Phys. Rev. B 40, 666 (1989).

[22] A. Checco, P. Guenoun, and J. Daillant, Phys. Rev. Lett. 91, 186101 (2003).

[23] L. Bruschi, A. Carlin, A. O. Parry, and G. Mistura, Phys. Rev. E 68, 021606 (2003).

[24] L. Bruschi, A. Carlin, and G. Mistura, Phys. Rev. Lett. 89, 166101 (2002).

[25] J. Als-Nielsen and D. McMorrow, Elements of Modern X-Ray Physics (John Wiley \& Sons, New York, 2001).

[26] C. T. Black, K. W. Guarini, K. R. Milkove, S. M. Baker, T. P. Russell, and M. T. Tuominen, Appl. Phys. Lett. 79, 409 (2001).

[27] O. Gang, M. Fukuto, P. Huber, and P. Pershan, Colloids Surf. A 206, 293 (2002).

[28] L. G. Parratt, Phys. Rev. 95, 359 (1954).

[29] J. N. Israelachvili, Intermolecular and Surface Forces: With Applications to Colloidal and Biological Systems (Academic Press, New York, 1992).

[30] V. Panella, R. Chiarello, and J. Krim, Phys. Rev. Lett. 76, 3606 (1996).

[31] A. O. Parry, J. M. Romero-Enrique, and A. Lazarides, Phys. Rev. Lett. 93, 086104 (2004). 